\title{
Time Resolved Photoemission to Unveil Electronic Coupling Between Absorbing and Transport Layers in a Quantum Dot Based Solar Cell
}

Charlie Gréboval ${ }^{1}$, Prachi Rastogi ${ }^{1}$, Junling Qu ${ }^{1}$, Audrey Chu ${ }^{1}$, Julien Ramade ${ }^{1}$, Adrien Khalili ${ }^{1}$, Corentin Dabard ${ }^{1}$, Tung Huu Dang ${ }^{1}$, Hervé Cruguel ${ }^{1}$, Abdelkarim Ouerghi ${ }^{2}$, Nadine Witkowski ${ }^{1}$, Mathieu G. Silly ${ }^{3}$, Emmanuel Lhuillier ${ }^{*}$

${ }^{1}$ Sorbonne Université, CNRS, Institut des NanoSciences de Paris, INSP, F-75005 Paris, France.

2 Université Paris-Saclay, CNRS, Centre de Nanosciences et de Nanotechnologies, 91120,

Palaiseau, France

${ }^{3}$ Synchrotron-SOLEIL, Saint-Aubin, BP48, F91192 Gif sur Yvette Cedex, France.

\begin{abstract}
Lead sulfide (PbS) colloidal quantum dots-based photodiodes are remarkable structures obtained via colloidal engineering because of their outstanding optoelectronic performances. They combine surface ligand engineering to design a $p$ - $n$ junction with all solution processability. Here we investigate the $\mathrm{PbS}$ diode electronic structure combining static and dynamic photoemissions with transport measurements. We show that the $n$-type nature of the $\mathrm{I}^{-}$capped PbS CQDs shifts the valence band away from the Fermi level compared to the thiol capped nanocrystals. This change in majority carriers can be probed using time resolved X-ray photoemission spectroscopy (TRXPS). We also prove that the photo-induced binding energy shift depends on the nanoparticle surface chemistry. Finally, we demonstrate the ability of TRXPS to selectively probe the electronic structure of each side of an interface. We explore the $\mathrm{PbS} / \mathrm{MoO}_{3}$ interface used as hole extractor in the $\mathrm{PbS}$ solar cell, using this method. We demonstrate that the $\mathrm{PbS}$ layer photosensitizes the $\mathrm{MoO}_{3}$ layer and that the two layers have a quasi-rigid electrostatic coupling. We identify the band bending occurring on the $\mathrm{PbS}(\mathrm{EDT}) / \mathrm{MoO}_{3}$ to be a limiting factor for the device performance and suggest strategies to overcome this limitation.
\end{abstract}

*To whom correspondence should be sent: el@insp.upmc.fr 


\section{Introduction}

Lead sulfide (PbS) colloidal quantum dot (CQD) based solar cells are certainly one of the greatest realization in colloidal engineering for optoelectronics. ${ }^{1}$ In a few years, the power conversion efficiency of this structure has raised from a few percent to $14 \% .^{2-4}$ The early concept was the use of nanocrystals with size-tunable absorption spectrum in order to match the optimal QueisserShockley band gap. It was simultaneously suggested that CQDs might be an interesting platform to use multi-exciton generation (MEG), thanks to the reduced power threshold of MEG in low dimensional materials. ${ }^{5}$ First devices were based on Schottky diodes ${ }^{6,7}$ and later, diverse concepts were introduced to enhance detection performance. In particular, halide passivation ${ }^{8,9}$ of the nanocrystals improved the material stability while enhancing the effective charge mobility. This concept proceeded with the development of halide-capped PbS nanocrystal inks ${ }^{10,11}$ which have efficiently enabled the fabrication of thick strongly photoconductive films.

In parallel, the rational design of a $p-n$ junction became possible ${ }^{12}$ thanks to systematic photoemission investigations of the band shift induced by series of capping ligands. ${ }^{13,14}$ These measurements provided a strategy to tune the carrier density in narrow band gap nanocrystals through ligand engineering. ${ }^{15-19}$ From the photonic point of view, the diode structure also enables multi passes ${ }^{20}$ of the light within the absorbing film. This also facilitates the realization of higher performance devices, with respect to planar photoconductive devices. ${ }^{21,22}$ The maturity of $\mathrm{PbS}$ CQDs based diodes also attracted interests beyond solar cell applications: for near IR passive and active imaging. ${ }^{14,23,24}$

Current best structure is based on the following stack ${ }^{25}$ ITO/ETL/PbS(n)/PbS(p)/HTL/Au, where ITO is tin-doped indium oxide used as transparent conductive layer, which is generally thick to reduce the contact resistance. The electron transport layer (ETL) is generally based on an oxide such as $\mathrm{TiO}_{2}$ or $\mathrm{ZnO}$ and contribute significantly to the rectifying behavior of the diode. PbS CQDs have a band gap around $950 \mathrm{~nm}$ corresponding to nanocrystals with $3.5 \mathrm{~nm}$ diameter. The $n$-type PbS layer is obtained by preparing an ink where the $\mathrm{PbS}$ is capped by $\mathrm{I}$. The $p$-type layer is obtained from the same nanocrystals, but processed with a solid-state ligand exchange by ethanedithiol (EDT) as capping ligands. A hole transport layer (HTL), generally based on $\mathrm{MoO}_{3}$, can be inserted and is covered by a gold metallic contact, whose role is to collect photogenerated holes and present air stable capping contact.

In this paper, we aim to discuss the role of the HTL layer on the electronic and transport properties of the PbS layer. Generally, the effect of this layer has been only probed via the transport and photodetection measurements (especially the open-circuit voltage). Here, we use X-ray photoemission spectroscopy (XPS) and time resolved X-ray photoemission spectroscopy ${ }^{26-35}$ (TRXPS) as a strategy to provide a consistent picture between the electronic spectrum and the transport properties. Moreover, the coupling between the hole collecting layer and the absorbing layer has been directly observed. In the long-time scale domain (i.e. above the transit time), we prove that the HTL spectrum shifts under illumination, whilst being excited below its absorbing band gap, due to its coupling to the absorbing layer. Furthermore, we show that the photo-induced shift follows the one of the PbS layer, suggesting that the electrostatic behavior of $\mathrm{MoO}_{3}$ is driven by the depletion width of $\mathrm{PbS}$. 


\section{METHODS}

Chemicals: Octadecene (ODE, Acros Organics, 90\%), lead oxide (PbO, Strem Chemicals, 99.999+\%-), oleic acid (OA, Alfa Aesar 90\%), hexamethyldisilathiane (TMS 2 S, Sigma Aldrich, synthesis grade), ethanol (VWR, >99.9\%), n-Octane (SDS, 99\%), n-Heptane (Merck, >99\%), nhexane (VWR, 99\%), toluene (Carlo Erba, >99.8\%), N,N-dimethylformamide (DMF, Sigma Aldrich), hydrochloric acid $\left(\mathrm{HCl}\right.$, Mieuxa, 25\%), ammonium iodide $\left(\mathrm{NH}_{4} \mathrm{l}\right.$, Alfa Aesar, $\left.\geq 99 \%\right)$, and 1,2 ethanedithiol (EDT, Fluka, 98\%)

$\mathrm{PbS}$ nanocrystal synthesis: The procedure is inspired from Hines et $\mathrm{al} \cdot{ }^{36} 0.9 \mathrm{~g}$ of $\mathrm{PbO}$ are introduced in a $100 \mathrm{~mL}$ three neck flask with $3 \mathrm{~g}$ of OA and $47 \mathrm{~g}$ of ODE. The flask is degassed under vacuum at $120^{\circ} \mathrm{C}$ for 2 hours. Meanwhile, in an air free glove box, a mixture of $420 \mu \mathrm{L}$ of TMS $\mathrm{T}_{2} \mathrm{~S}$ and $10 \mathrm{~mL}$ of ODE is prepared in a $20 \mathrm{~mL}$ vial and then introduced into a $20 \mathrm{~mL}$ syringe. The atmosphere of the flask is switched to argon ( $\mathrm{Ar}$ ) and the temperature is set to $90^{\circ} \mathrm{C}$. The $\mathrm{TMS}_{2} \mathrm{~S}$ solution is quickly injected, and the solution turns into dark color while the temperature drops to $80^{\circ} \mathrm{C}$. After $8 \mathrm{~min}$ at $80^{\circ} \mathrm{C}$, the reaction is stopped by removing the heating mantle and prompt cooling of the flask by addition of a mixture of heptane and OA. The nanoparticles are then precipitated by addition of ethanol. The formed pellet is redispersed in toluene. A second step of cleaning is repeated. Finally, the pellet is redispersed in toluene with a $50 \mathrm{mg} \mathrm{mL}^{-1}$ concentration. The solution is centrifuged to remove any colloidally unstable materials. Finally, the solution is filtered on a $0.22 \mu \mathrm{m}$ PTFE filter.

PbS Ink preparation: A solution of $\mathrm{NH}_{4} \mathrm{l}$ at $60 \mathrm{mg} \mathrm{mL}^{-1}$ in DMF is prepared by sonication. $2.5 \mathrm{~mL}$ of this solution is introduced in a test tube. On the top of this polar phase, $2.5 \mathrm{~mL}$ of PbS nanocrystal solution at $50 \mathrm{mg} \mathrm{mL}^{-1}$ in toluene is deposited and diluted with hexane. The two phases are then mixed and a phase transfer is observed. The clear non-polar phase is discarded and the DMF solution is cleaned three more times using hexane. Finally, the particles are precipitated by introducing ethanol in the tube. After centrifugation, the formed pellet is redispersed in fresh DMF with a concentration of $250 \mathrm{mg} \mathrm{mL}^{-1}$. The solution is finally centrifuged to discard any unstable materials.

Solid state ligand exchange: A solution at $1 \%$ in mass of EDT in ethanol is prepared. PbS nanocrystals solution in hexane:octane (9:1 volume ratio) is drop-casted onto the electrodes and left to dry. The resultant thin film is dipped in the ligand solution and then rinsed with acetone. Once the film is dried, a second layer of PbS nanocrystals is deposited and the ligand exchange step is repeated. The deposition process is performed two times in total.

ITO patterning: We start from commercial glass/ITO substrates of $30 \times 30 \mathrm{~mm}^{2}$ with a low sheet resistance $(7 \Omega / \square)$. Substrates are cut into $15 \times 15 \mathrm{~mm}^{2}$ pieces and cleaned by sonication in an acetone bath for $5 \mathrm{~min}$. The cleaning procedure is followed by rinsing with acetone and iso-propanol the substrates that are then dried with dry $\mathrm{N}_{2}$ flow. AZ 5214E photoresist is spin-coated for $30 \mathrm{~s}$, then baked at $110^{\circ} \mathrm{C}$ for $90 \mathrm{~s}$. A standard photolithography process is performed using mask aligner for exposing the substrates to UV light for $5 \mathrm{~s}$ through a lithography mask ( $1 \mathrm{~mm}$ width). Photoresist is developed using AZ 726 developer for $20 \mathrm{~s}$ and immediately rinsed with deionized water. After that, exposed ITO surface is completely etched out with $25 \% \mathrm{HCl}$ (in water) for $15 \mathrm{~min}$ at $40^{\circ} \mathrm{C}$, and substrates are immediately dipped into deionized water. Then, we conduct lift-off process in an acetone bath and patterned ITO substrates are cleaned with acetone and isopropanol. Finally, substrates are dried with dry $\mathrm{N}_{2}$ flow.

$\mathrm{TiO}_{2}$ film preparation: $\mathrm{TiO}_{2}$ films are prepared from a commercial solution (SOLARONIX Ti-Nanoxide $\mathrm{HT}$-L/SC). The solution is dropped on heated patterned ITO $\left(110^{\circ} \mathrm{C}\right)$ and spin-coated at $5000 \mathrm{rpm}$ 
during $30 \mathrm{~s}$. The pre-heating process is required to improve the solution wetting. Resultant ITO/TiO2 sample is then heated at $450{ }^{\circ} \mathrm{C}$ for $15 \mathrm{~min}$ and its thickness is measured to be $120 \mathrm{~nm}$ with DEKTAK profilometer.

n-doped PbS ink deposition: A $250 \mathrm{mg} \mathrm{mL}^{-1}$ solution of $n$-doped $\mathrm{PbS}$ ink is used for the active material layer. $60 \mu \mathrm{L}$ of this solution is deposited on the ITO/TiO 2 substrate and spin-coated at 1000 rpm for $60 \mathrm{~s}$, followed by $2000 \mathrm{rpm}$ for $240 \mathrm{~s}$. The procedure is repeated twice. Depositions are performed in air-free environment (ie in $\mathrm{N}_{2}$ filled glove box).

p-doped PbS: $60 \mu \mathrm{L}$ of a $30 \mathrm{mg} \mathrm{mL}^{-1}$ of OA capped $\mathrm{PbS}$ in toluene is deposited on the ITO/TiO $/ \mathrm{n}$ doped PbS substrate and spin-coated at $2000 \mathrm{rpm}$ for $30 \mathrm{~s}$. A solid-state ligand exchange (see above) is then performed. This procedure is repeated twice. Depositions are performed in air-free environment.

$\mathrm{MoO}_{3} / \mathrm{Au}$ deposition: After the deposition of PbS layers, the device is transferred from a glovebox to an evaporator while keeping an air-free environment. After this transfer and once a vacuum around $5.10^{-6}$ mbar is reached, $10 \mathrm{~nm}$ of $\mathrm{MoO}_{3}$ followed by $80 \mathrm{~nm}$ of gold are evaporated. The sample is then stored in air-free environment.

Infrared absorption: For infrared spectroscopy, we use a Fischer Nicolet iS50 FTIR in either Attenuated Total Reflection (ATR) or in transmission configuration. The spectra are averaged over 32 acquisitions and have a $4 \mathrm{~cm}^{-1}$ resolution.

UV-visible absorption: For UV-visible spectroscopy, we use a JASCO V-730 spectrometer.

The nanocrystal composition is studied thanks to Energy Dispersive $X$-ray spectroscopy. The latter is conducted in a scanning electron microscope (Zeiss Supra 40) operated at $5 \mathrm{kV}$. A Bruker probe (EDS Compact $30 \mathrm{~mm}$ ) is used to study the energy of the scattered electron.

For electron microscopy imaging, a diluted solution of nanocrystal is drop-casted on a copper grid with on top of which an amorphous carbon layer. The grid is degassed overnight under secondary vacuum. Transmission electron microscopy (TEM) measurement was performed with a JEOL 2010F. For scanning electron microscopy (SEM) we use FEI Magellan microscope operated under low bias $(3 \mathrm{kV})$ and low current $(6 \mathrm{pA})$ to avoid charging effect

For film thickness determination, we used a Dektak 150 Veeco profilometer and FEI Magellan scanning electron microscope.

$X$-ray photoemission measurements (XPS): Photoemission spectroscopy has been conducted on the Tempo beamline of synchrotron Soleil. Films of nanocrystals are spin-casted on a gold coated Si substrate (gold layer is $80 \mathrm{~nm}$ thick). The ligands of the nanocrystals are exchanged using the previously described procedures. ${ }^{9,10}$ Samples are introduced in the preparation chamber and degassed until vacuum below $10^{-9}$ mbar is reached prior to their introduction into the analysis chamber. A $600 \mathrm{eV}$ photon energy is typically used for core level analysis, while a lower energy of $150 \mathrm{eV}$ is used for valence band and secondary electron cut-off. The signal is acquired onto a Scienta Ses2002 electron analyzer equipped with a delay line detector developed by Elettra. ${ }^{27}$ Acquisition is done at constant pass energy within the detector. Core levels are acquired with a 50 $\mathrm{eV}$ pass energy, while time resolved measurements are conducted with a $200 \mathrm{eV}$ pass energy. A gold substrate is used to calibrate the Fermi energy. The absolute value of the incoming photon energy is determined by measuring the second order of $\mathrm{Pb}$ core level peaks. Low binding energy 
part of the photoemission spectrum is used to determine the value of the valence band maximum with respect to the Fermi level, $\mathrm{V}_{\mathrm{B}}-\mathrm{E}_{\mathrm{F}}$. Then the energy of the conduction band is determined from the valence band offset of the absorption gap. Finally, the work function is determined by measuring the cut-off of secondary electrons.

Time resolved $X$-ray photoemission measurements (TRXPS): The sample is excited using a $635 \mathrm{~nm}$ laser diode which is electrically chopped. The frequency of the laser is matched to capture the long decay time of the surface photovoltage signal, typically here using a $100 \mathrm{~Hz}$ frequency. We use a $40 \%$ duty cycle (ie laser is on for $4 \mathrm{~ms}$ and off for $6 \mathrm{~ms}$ ). Meanwhile a photoemission spectrum is acquired every $10 \mu \mathrm{s}$, note that the photoelectron analyzer resolution is $30 \mathrm{~ns}$ (limited by the spread of the electrons between the hemispheres) far below the targeted acquisition time. In usual time resolved experiments based on core level spectrum measurement for different temporal delays, the energy determination of the different contributions of core level spectra depends on energy stability and accuracy of the power supply which is about $10-20 \mathrm{meV}$, sample modification (aging), beamline energy stability at long temporal range, and the statistic of the measured spectrum. In stroboscopic time resolved measurement performed in fixed mode, as the cyclic measurement is averaged for a long period compared to the time dynamics of the measured physical phenomenon, all the above variations lead to a broadening of the spectra but not to a kinetic energy shift. Core level energy positioning is only limited by statistics leading to few meV energy resolution, the energy uncertainty being determined by the fit uncertainty.

Electrodes fabrication for transistor: A glass substrate is first rinsed and then sonicated in acetone for $5 \mathrm{~min}$. The wafer is then rinsed with acetone and isopropanol and finally is conducted by plasma cleaning for $5 \mathrm{~min}$. Then AZ 5214E resist is spin coated on the substrate and baked at $110^{\circ} \mathrm{C}$ for $90 \mathrm{~s}$. The resist is exposed through a shadow mask to UV illumination for $2 \mathrm{~s}$, with black paper below the substrate. The resist is baked again at $125^{\circ} \mathrm{C}$ for $2 \mathrm{~min}$ and re-exposed to UV (without the mask) for $40 \mathrm{~s}$. The resist is then developed using AZ726. The film is rinsed with water and finally dried. The remaining organic layer is removed by plasma cleaning. $5 \mathrm{~nm}$ of chromium and $80 \mathrm{~nm}$ of gold are evaporated and the remaining resist is removed by immersing the film in acetone for one hour. The electrodes are then rinsed with isopropanol and finally dried. The interdigitated electrodes include 25 digits, each $2.5 \mathrm{~mm}$ long with a $20 \mu \mathrm{m}$ gap between them. The optical area of the device is $0.037 \mathrm{~cm}^{2}$.

Electrolyte gating: For electrolyte gating we first mixed in a glove box $0.5 \mathrm{~g}$ of $\mathrm{LiClO}_{4}$ with $2.3 \mathrm{~g}$ of PEG $\left(\mathrm{Mw}=6 \mathrm{~kg} \cdot \mathrm{mol}^{-1}\right)$. The vial is heated at $170{ }^{\circ} \mathrm{C}$ on a hot plate for $2 \mathrm{~h}$ until the solution turns clear. The electrolyte solution is warmed to around $100^{\circ} \mathrm{C}$ and brushed on the top of the $\mathrm{PbS}$ film.

Transistor measurements: The sample is connected to a Keithley 2634B which sets the drain bias, controls the gate bias ( $\mathrm{V}_{\mathrm{GS}}$ ) with a step of $1 \mathrm{mV}$ and measures the associated currents IDs and IGs. All measurements are done in air at room temperature. 


\section{Results and discussion}

In order to probe interfaces between the absorbing and the transport layers which will be relevant with the state of the art of devices, we start by building a PbS CQD-based solar cell. We synthesize PbS CQDs according to the method proposed by Hines et al. ${ }^{36}$ The obtained CQDs have typically a diameter $3.5 \pm 1 \mathrm{~nm}$ and present an excitonic peak in their absorption spectrum at $940 \mathrm{~nm}$, see Figure 1a. Energy dispersive X-ray spectroscopy reveals an excess of lead ${ }^{37}(\mathrm{~Pb}: \mathrm{S}$ atomic ratio is found to be 1.37). This native non stoichiometry leads to an inherent doping for the particles, which can be further tuned by introducing surface dipoles thanks to ligand exchange ${ }^{13}$.

We then pattern an ITO substrate and coat it with a compact $\mathrm{TiO}_{2}$ layer which is sintered at high temperature $\left(450^{\circ} \mathrm{C}\right)$ to improve its conductivity. Out of the PbS CQDs, we prepare an ink in which the CQDs are finally capped with I- ions using the phase transfer approach. ${ }^{38}$ This ink is spin-coated on the $\mathrm{TiO}_{2}$ layer forming $\mathrm{a} \approx 200 \mathrm{~nm}$ thick layer. On top of this layer, PbS CQDs dispersed in toluene and capped with their long native ligands (oleic acid, see figure S1) are spin-coated and a ligand exchange is performed toward EDT ligands. The absoprtion band gap of this layer is marginally affected by this process with a small redshift of the order of $20 \mathrm{meV}$ as compared to the layer capped with iodine, see figure S2. Two layers of the EDT capped PbS CQDs are deposited to form a shortfree layer. The deposition steps relative to the $\mathrm{PbS} C Q D$ s are conducted in an air-free glove box. Finally using a thermal evaporator and an air free transfer, a first thin (10 nm) layer of $\mathrm{MoO}_{3}$ is evaporated, followed by the evaporation of $80 \mathrm{~nm}$ of gold. SEM image of the obtained device is provided in Figure 1b. The electrical characteristics of the resulting device are shown in Figure 1c. The obtained I-V curves display a clear rectifying behavior and a strong current modulation under illumination. The open circuit voltage is larger than $0.5 \mathrm{~V}$ under a $940 \mathrm{~nm}$ monochromatic illumination with an irradiance of $56 \mathrm{~mW} \mathrm{~cm}^{-2}$.
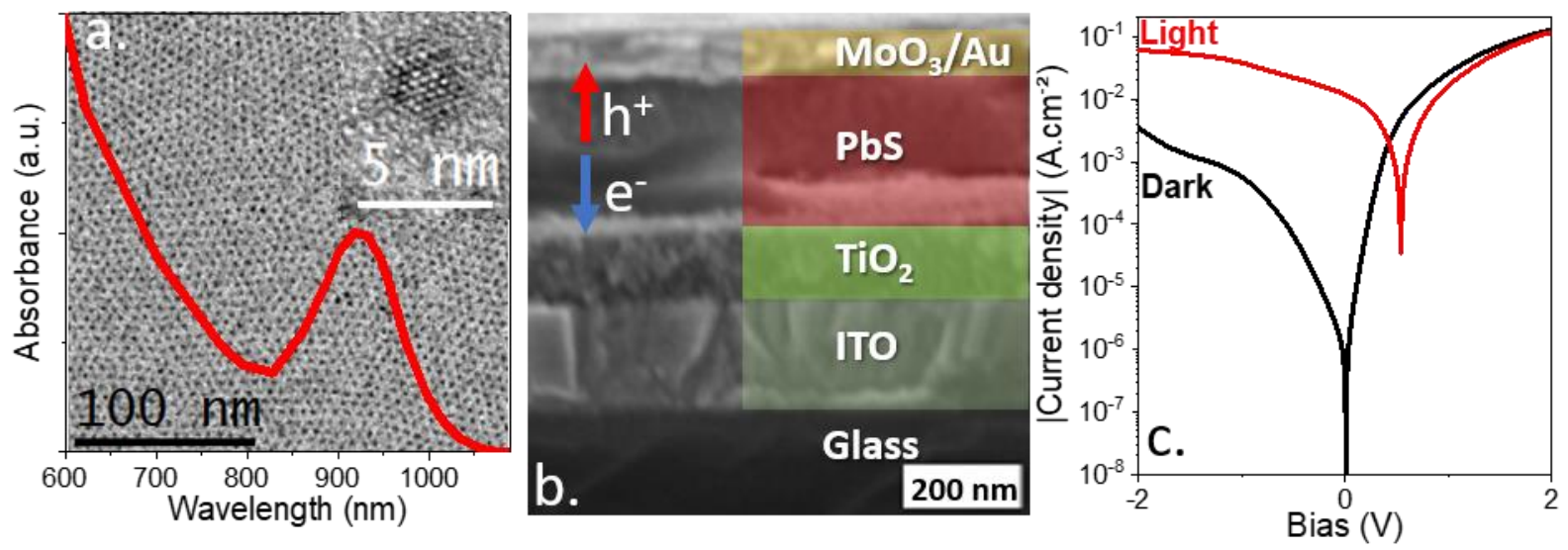

Figure 1: a. Absorption spectrum of the PbS nanocrystals used for the fabrication of the photodiode. The background is a TEM image of the PbS CQDs. The top inset is an high resolution TEM image of the PbS NC. b. SEM image of the PbS photodiode. On the top of the glass substrate, four layers can be distinguished: the ITO transparent conductive layer, the n-type $\mathrm{TiO}_{2}$ layer, the absorbing layer consisting of PbS CQDs and finally the top contact made of $\mathrm{MoO}_{3}$ and gold. c. I-V curves of the photodiode in the dark and under illumination by a $940 \mathrm{~nm}$ laser diode $\left(56 \mathrm{~mW} \mathrm{~cm}^{-2}\right)$

In the following, we build a consistent picture of the static and dynamic electronic structure of the active layers and correlate them to their transport properties. To do so, each layer has been studied independently. The XPS spectrum of the PbS layer is given in Figure $2 \mathrm{a}$. Clear constributions of $\mathrm{Pb}$ and $\mathrm{S}$ atoms have been observed. In particular, the analysis of the core levels is given in Figure $2 \mathrm{~b}$, 
S3-S5. The $\mathrm{Pb} 4 \mathrm{f}$ core level presents two contributions (see Figure S5) which can be attributed to the lead coupled to the sulfide for the main contribution (binding energy of $137.76 \mathrm{eV}$ ) and the lead coupled to oxide for the highest energy contribution (binding energy of $138.76 \mathrm{eV}$ ). This suggests a partial oxidation on the CQD surface. This has to be correlated with the presence of a small oxygen contribution in the XPS overview coming from $\mathrm{O} 1 \mathrm{~s}$, even though this may partly come from the leftover of oleic acid ligands. The surface chemistry clearly affects the binding energy of the $\mathrm{Pb} 4 \mathrm{f}$ state, see Figure 2b. As ligands are switched from EDT to $\mathrm{I}^{-}$, the binding energy of the $\mathrm{Pb} 4 \mathrm{f}$ state and S $2 p$ state (Figure S5) increases suggesting the presence of a dipole resulting from the capping of the $\mathrm{PbS}$ by $\mathrm{I}^{-}$ions. The magnitude of the shift is nevertheless higher for $\mathrm{Pb}$ than for $\mathrm{S}$ as previously observed. ${ }^{39}$ In order to give a complete picture of the band alignment that is of crucial importance for the optoelectronic properties of the $\mathrm{PbS}$ layer, we investigate the valence states in the vicinity of the Fermi level, see Figure $2 c$ and $d$. In Figure 2e, a reconstructed energy spectrum in absolute energy scale (ie vs vacuum) is proposed. The energy of the valence band is extracted from the low energy part of the photoemission spectrum. The energy of the conduction band is determined relatively to the valence band through the optical band gap. Finally the vacuum state is determined with respect to the Fermi level thanks to the cut-off of the secondary electrons.

Table 1: Optical band gap, work function $\left(W_{F}\right)$ and valence band onset $\left(V_{B}-E_{F}\right)$ deduced from experimental data for PbS CQDs capped with $\mathrm{P}^{-}\left(\mathrm{PbS} \mathrm{NH}_{4} \mathrm{l}\right)$, PbS CQDs capped with EDT (PbS EDT) and at the interface with $\mathrm{MoO}_{3}\left(\mathrm{PbS} E D T-\mathrm{MoO}_{3}\right)$

\begin{tabular}{llll}
\hline Sample & $\begin{array}{l}\text { Optical } \\
\text { Band gap } \\
(\mathrm{eV})\end{array}$ & $\mathrm{W}_{\mathrm{F}(\mathrm{eV})}$ & $\begin{array}{l}\mathrm{E}_{\mathrm{F}-\mathrm{V}_{\mathrm{B}}} \\
(\mathrm{eV})\end{array}$ \\
\hline $\mathrm{PbS} \mathrm{NH}$ I & 1.32 & 4.91 & 0.72 \\
\hline $\mathrm{PbS}$ EDT & 1.30 & 4.31 & 0.47 \\
\hline $\begin{array}{l}\mathrm{PbS} \mathrm{EDT}_{\mathrm{MoO}} \\
\mathrm{MOO}_{3}\end{array}$ & 1.30 & 4.41 & 0.33 \\
\hline
\end{tabular}

As noticed from Table 1, the optical gap is mostly not affected by the nature of the capping of PbS CQDs confirming the similarity in size and crystallinity for the two types of PbS CQDs. ${ }^{40} \mathrm{~A}$ major difference is rather observed in the work function for the two CQD capping that originates from the domination of the surface dipole contribution in the case of $\mathrm{I}^{-}$capping that is not balanced by liganddipole moment contribution as already observed on such CQDs. ${ }^{13,14}$ The nature of the majority carriers is given by the valence band onset $\left(V_{B}-E_{F}\right)$ from Table 1 that shows a slight $n$-type nature $\left(V_{b}-E_{F}>E_{G} / 2\right)$ for $I^{-}$capping and a p-type nature $\left(V_{b}-E_{F}<E_{G} / 2\right)$ for the EDT capping that is even more pronounced at the interface with $\mathrm{MoO}_{3}$, see Figure $2 \mathrm{e}$ and $\mathrm{S} 4-5$. The semiconductor nature $(n$ or $p)$ is further confirmed by surface photovoltage experiments as described in the following of the paper. 

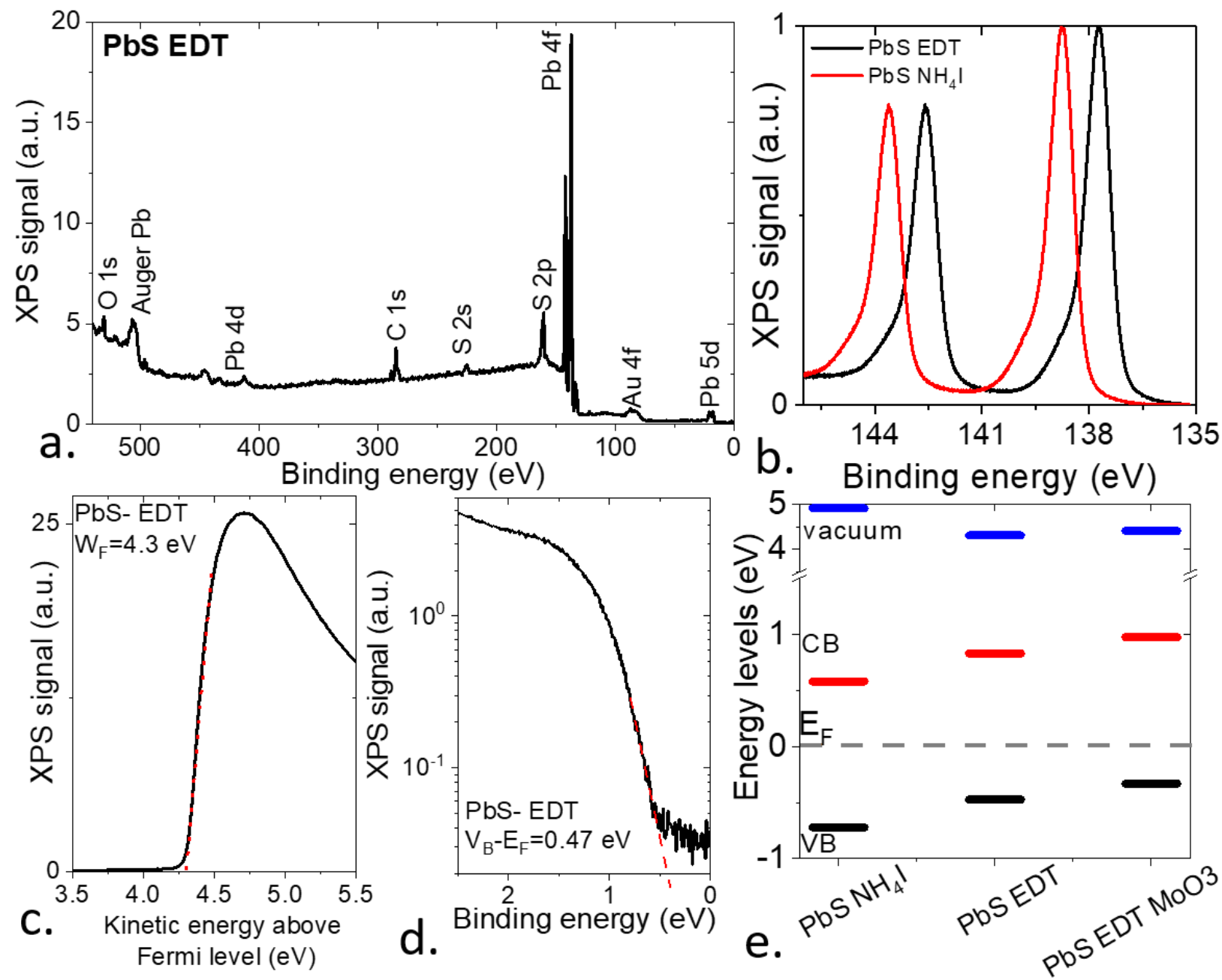

Figure 2: a. X-ray photoemission overview spectrum relative to a thin film of EDT capped PbS CQDs. b. Photoemission spectra relative to the Pb $4 f$ state for PbS CQDs capped with EDT and $\mathrm{r}$. Also see Figure S3 and S4. c. Photoemission spectrum relative to the cut-off of the secondary electrons for a thin film made of PbS CQDs capped with EDT. $d$. Photoemission spectrum relative to the valence band for a thin film consisting of PbS CQDs capped with EDT. e. Electronic spectrum in absolute energy scale for PbS CQDs capped with I-, capped with EDT and capped with EDT topped by a layer of $\mathrm{MoO}_{3}$. The Fermi energy is set equal to zero. The black, red, and blue lines are respectively the valence band, the conduction band, and the vacuum level.

In the next step, TRXPS, a pump probe method, ${ }^{41}$ is used, see Figure $3 \mathrm{a}$, to study carrier dynamics in the CQDs and at the interface with HTL. The sample is first excited by a laser $(635 \mathrm{~nm}$ or $\approx 2 \mathrm{eV}$ energy for the photon) to excite the PbS CQDs above their band gap $(1.32 \mathrm{eV})$ and generates an exciton. Then the photoemission is used as a probe. A series of photoemission spectra is acquired during and after illumination and we then follow the energy of each peak by making them fit to Gaussian models and averaged cyclically until suitable statistic. Such protocol leads to an energy resolution of $\approx$ few $\mathrm{meV}$ below the one of static XPS method $(\approx 50 \mathrm{meV})$ as detailed in the method section A typical time trace of the $\mathrm{Pb} 4 \mathrm{f}$ state obtained from $\mathrm{PbS}$ CQDs is given in Figure $3 \mathrm{~b}$. The process occurring in the semiconductor is explained in Figure $3 \mathrm{c}$ when the light is turned on and in Figure $3 d$ when the excitation is shut down. The principle of work in the non-contact surface photovoltage provided with TRXPS is the following. In a first step, the pump, under illumination, generates photocarriers. The minority carriers flow toward the surface and are accumulated at the interface which little by little reduces the band bending toward the flat band condition. ${ }^{41}$ Note that, here the time scale has been chosen to capture the long component of the TRXPS signal and so 
the fast rise of the signal under illumination is not resolved. When the pump is turned off, the majority carriers from the bulk of the sample flow toward the accumulated minority carriers to recover neutrality and the two carriers are recombined, restoring the initial band bending.

The typical range of power used for the laser corresponds to an irradiance $(I)$ of $\approx 14 \mathrm{~W} . \mathrm{cm}^{-2}$. The photoinduced carrier density is given by $n_{\text {light }}=\frac{I \lambda \sigma \tau}{h c}$ with $\lambda$ the excitation wavelength $(635 \mathrm{~nm}), \sigma$ the absorption cross section (in the $2-6 \times 10^{-16} \mathrm{~cm}^{-2}$ range according to literature ${ }^{42,43}$ ), $\tau$ the carrier lifetime taken equal to $2 \mu \mathrm{s},{ }^{37} h$ the Planck's constant and $c$ the speed of light, leading to $n \approx 0.04<<1$ carrier per CQD. It is thus reasonable to exclude any coulombic effect which may result from multiexciton charging in the following analysis of the TRXPS data.
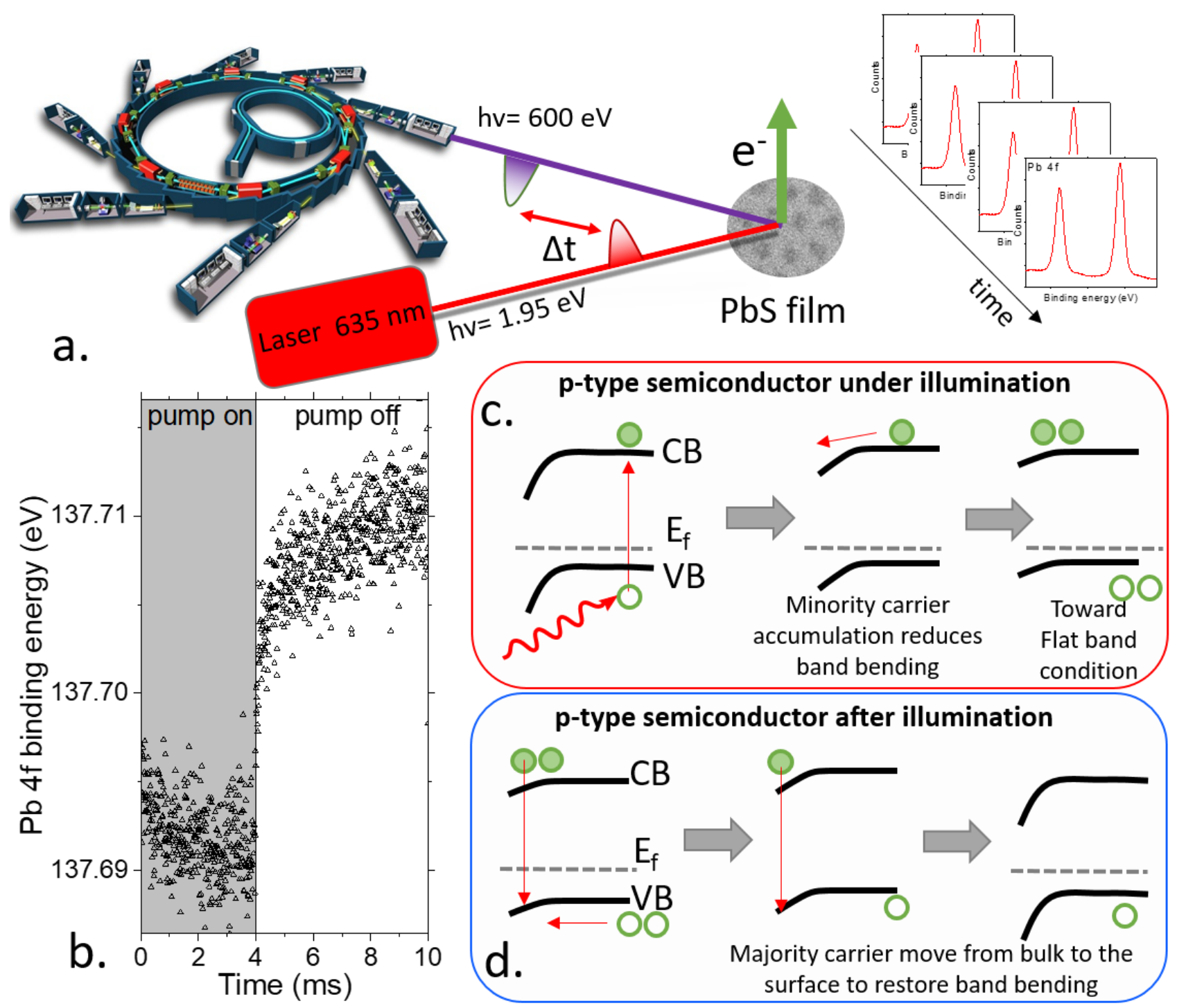

Figure 3: a. Scheme of the pump-probe photoemission experiment. A laser diode at $635 \mathrm{~nm}$ is used to excite the PbS CQDs film, while the probe is the synchrotron beam at $600 \mathrm{eV}$. Photoemission of the $\mathrm{Pb} 4 \mathrm{f}$ state is then followed as a function of time. $b$. Energy of the Pb $4 \mathrm{f}$ state from a EDT capped $\mathrm{PbS} C Q D$ s film as a function of time, as the laser light at $635 \mathrm{~nm}$ is turned on and off. c. Scheme of the band bending occuring in a p-type semiconductor film under illumination. $d$. Scheme of the band bending occuring in a p-type semiconductor film after turning-off the illumination. The figure is adapted with permission from ref ${ }^{32}$. Copyright (2020) American Chemical Society.

Beyond the energy resolution of the TRXPS, two key information can be extracted: (I) the nature of the majority carriers (i.e. hole vs electron) and (ii) the transport dynamics of the majority carriers 
(turn-off time). Using TRXPS, we probed a layer of PbS CQDs capped with I' and EDT ligands, see Figure $4 \mathrm{a}$ and $\mathrm{c}$. The sign of the photo-induced shift is changed for the two ligands: I- leads to a positive shift (upward band bending) while the thiol results in a negative shift (downward band bending). Such opposite signature suggests different majority carriers depending on ligands: electrons for $\mathrm{I}^{-}$and holes for EDT. This picture is fully coherent with static photoemission, where the thiol capped CQDs have a stronger $p$-nature (i.e. reduced value of $\mathrm{V}_{\mathrm{B}}-\mathrm{E}_{\mathrm{F}}$ ). To build a consistent picture of the sample, we used a layer of PbS CQDs as a channel of a field-effect transistor; see the inset of Figure $4 \mathrm{~b}$ for a scheme of the device. Here, we used an electrolytic transistor where the gate effect results from the ion displacement in an ion-gel matrix. ${ }^{44}$ Compared to conventional dielectric gating, this method presents several advantages such as (i) a much larger gate capacitance ( $\approx 1 \mu \mathrm{F} \mathrm{cm}{ }^{-2}$ compared to $10 \mathrm{nF} . \mathrm{cm}^{-2}$ for a $400 \mathrm{~nm}$ thick layer of $\mathrm{SiO}_{2}$ ) and (ii) stable transport measurement in air.
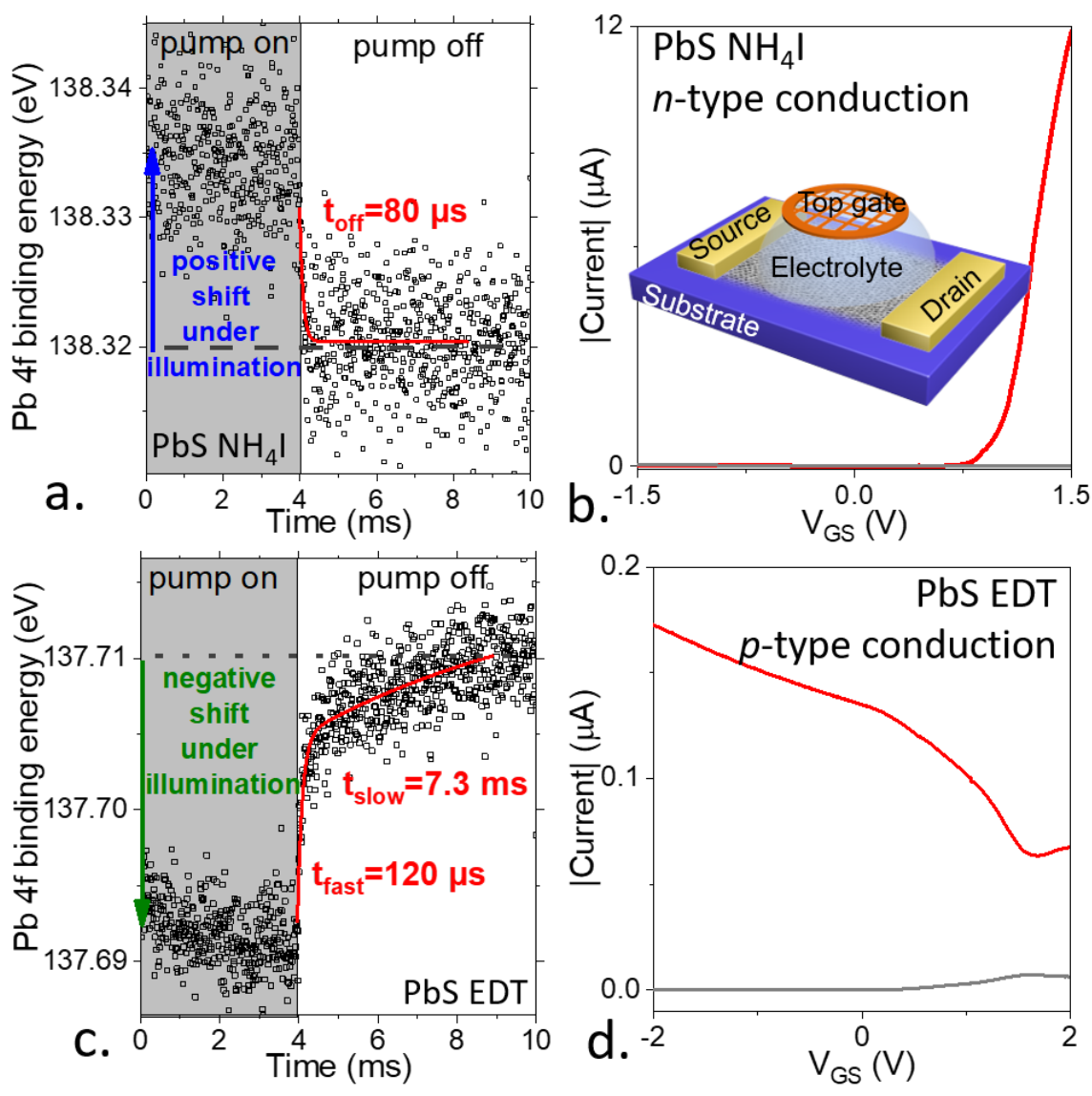

Figure 4: a. (resp. c.) Binding energy of the Pb 4f state from an I- capped (resp. EDT capped) PbS CQD film as a function of time, as the laser light at $635 \mathrm{~nm}$ is turned on and off. b.(resp. d.) Transfer curves (drain current in red and gate current in grey as a function of the applied gate bias) for a transistor, whose channel is made of l- capped (resp. EDT capped) PbS CQD film. The drain source is set equal to $400 \mathrm{mV}$. The inset of part $b$. is a scheme of the electrolytic transistor.

In the case of $\mathrm{I}^{-}$capping, we observe a rise in the channel conduction under electron injection (i.e. positive gate bias, see Figure $4 \mathrm{~b}$ ) which is the signature of an $n$-type semiconductor. At the opposite, the conductance of the thiol capped PbS CQDs channel increases under hole injection (i.e. negative gate bias see Figure $4 d$ ), verifying its $p$-type nature. $., 13,20$ Thus, we have obtained a unified picture between static, dynamic photoemissions, and transport measurements regarding the electronic structure of the active layer. Concerning the dynamics, we have measured a TRXPS relaxation time slower in the case of EDT (two components of $120 \mu \mathrm{s}$ and $7 \mathrm{~ms}$ ) capping compared to I' passivation (80 $\mu \mathrm{s}$ for the decay time). This observation may be attributed to a difference of carrier mobility between the electron and the hole as majority carriers switches while ligands get exchanged. 
In this last part, we take advantage of the atomic selectivity of photoemission. TRXPS allows the probing of atom dynamics on each side of an interface, as long as the top layer is thin enough compared to the photoelectron escaping depth. The coupling of lead chalcogenide layers to electron transport layers has previously been studied, ${ }^{45,46}$ including by TRXPS ${ }^{30}$. On the other hand, far less work has been devoted to the hole side. Here, we apply this method to the $\mathrm{PbS} / \mathrm{MoO}_{3}$ interface. We first investigate the TRXPS signal from the pristine $\mathrm{MoO}_{3}$ layer. The band gap of the $\mathrm{MoO}_{3}$ layer $(3.5 \mathrm{eV})$ being much larger than the photon energy of the pump, we expect no pump absorption, see Figure 5a. And consequently, no shift of the TRXPS signal is observed, see Figure 5b.

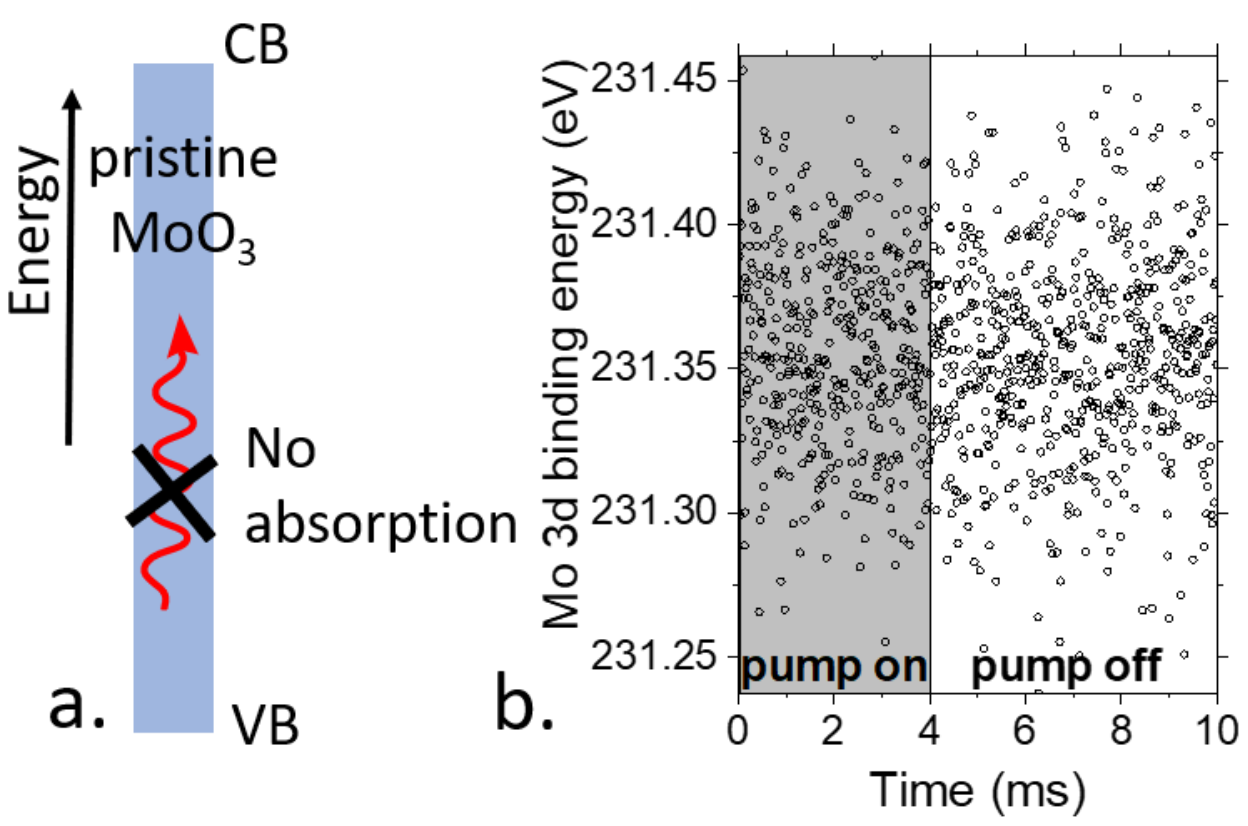

Figure 5: a. Scheme of the electronic spectrum of $\mathrm{MoO}_{3}$ excited by a photon at $635 \mathrm{~nm}$ (i.e. below band gap) where no absorption occurs. b. Binding energy of the $\mathrm{Mo} 3 \mathrm{~d}$ state from a $\mathrm{MoO}_{3}$ thin film as a function of time, as the laser light at $635 \mathrm{~nm}$ is turned on and off.

We then conduct the same experiment for a $\mathrm{MoO}_{3}$ layer deposited on the top of an EDT treated $\mathrm{PbS}$ layer, see Figure 6 and S6-7. In this case, we expect the PbS layer to be excited by the pump and generates electron hole pairs. For the pristine $\mathrm{PbS}$, the electronic structure and its band bending, characteristic of a p-type semiconductor, are depicted in Figure 6a. Once the $\mathrm{MoO}_{3}$ is deposited (Figure $6 \mathrm{~b}-\mathrm{c}$ ), we can still follow the signal coming from the $\mathrm{Pb} 4 \mathrm{f}$ state, see Figure $6 \mathrm{~d}$, because the $\mathrm{MoO}_{3}$ layer is thin $(<10 \mathrm{~nm})$ and discontinuous. The sign (negative) and magnitude of the shift (25 meV compared to $20 \mathrm{meV}$ ) remain similar to the case of the pristine PbS EDT capped layer shown in Figure 4c On the other hand, the signal of the Mo $3 \mathrm{~d}$ is drastically influenced since a negative shift toward Fermi level of about $35 \mathrm{meV}$ is now observed, see Figure 6e. It is interesting to note that, within the resolution of this experiment, the dynamics of the two layers appears similar, with a fast component of the order of $100 \mu \mathrm{s}$, suggesting a full electrostatic coupling of the two layers, see figure S8.

Possible mechanisms underlying this observation can be foreseen. First, for both $\mathrm{Pb}$ and $\mathrm{Mo}$ core levels, negative shift is observed under illumination which is consistent with positive charge carrier in both the CQDs and the HTL layer as expected. Because, the shift toward Fermi energy on Mo 3d is permanent upon illumination and toward Fermi level it does not reflect a possible hole charge transfer that could occur between the CQDs and the HTL ${ }^{47}$ layer. More likely, this behavior reflects the charge accumulation in the oxide at the oxide/ CQDs interface. Such accumulation is the result 
of the down ward band bending observed for both pristine and $\mathrm{MoO}_{3}$ functionalized $\mathrm{PbS}$ EDT. Such bending leads to the formation of a Schottky barrier at the interface with the top contact. ${ }^{48}$

The depletion width at the metal insulator interface is given by $L_{D}=\sqrt{\frac{2 \varepsilon \Delta V}{e p}}$ with $\varepsilon$ the dielectric constant, $\Delta V$ the height of the barrier (20 -35 mV according to TRXPS), $e$ the proton charge and $p$ the carrier density. The latter can be determined from the relative position of the Fermi level with respect to the valence band. For the $\mathrm{EDT} / \mathrm{MoO}_{3}$ sample we find the Fermi level to be $0.33 \mathrm{eV}$ above. This corresponds to a hole carrier density $p=8 . N$. $\exp \left(-0.33 e / k_{b} T\right)$ with $N$ the CQD density and the factor 8 corresponds to the band degeneracy in PbS. Assuming a randomly close pack film, $N$ is given by $0.64 /\left(4 / 3 \pi R^{3}\right)$ with $R$ the particle radius $\left.1.75 \mathrm{~nm}\right), N$ is $3 \times 10^{19} \mathrm{~cm}^{-3}$. This leads to $p=6.610^{14}$ holes. $\mathrm{cm}^{-3}$ and finally to $L_{D}=0.2 \mu \mathrm{m}$. This value is typically ten times larger than the thickness of the PbS EDT layer $(20 \mathrm{~nm})$. It is thus reasonable to assume that the whole layer is affected by the band bending.

Photogenerated holes get accumulated in the PbS-EDT layer, instead of being collected on the gold contact. Even if the barrier is reduced under illumination due to the flattening of the band, this situation is far less favorable than the one observed for perovskite nanocrystal ${ }^{32}$ where the interface with $\mathrm{MoO}_{3}$ leads to an opposite band bending facilitating the photohole extraction. This unfavorable band bending explains part of the $\mathrm{V}_{\mathrm{oc}}$ loss observed in $\mathrm{PbS}$ solar cell ${ }^{49,50}$ compared to perovskite material. Thus, the EDT capped PbS layer is very favorable to induce the pn junction within the absorbing layer but is suboptimal for charge extraction. Future design of the photodiode will have to include strategies to reduce this barrier for photohole. This might be obtained from a self-assembled monolayer inducing dipole ${ }^{51}$ or by using graded band gap CQD layer ${ }^{52}$ at this contact.

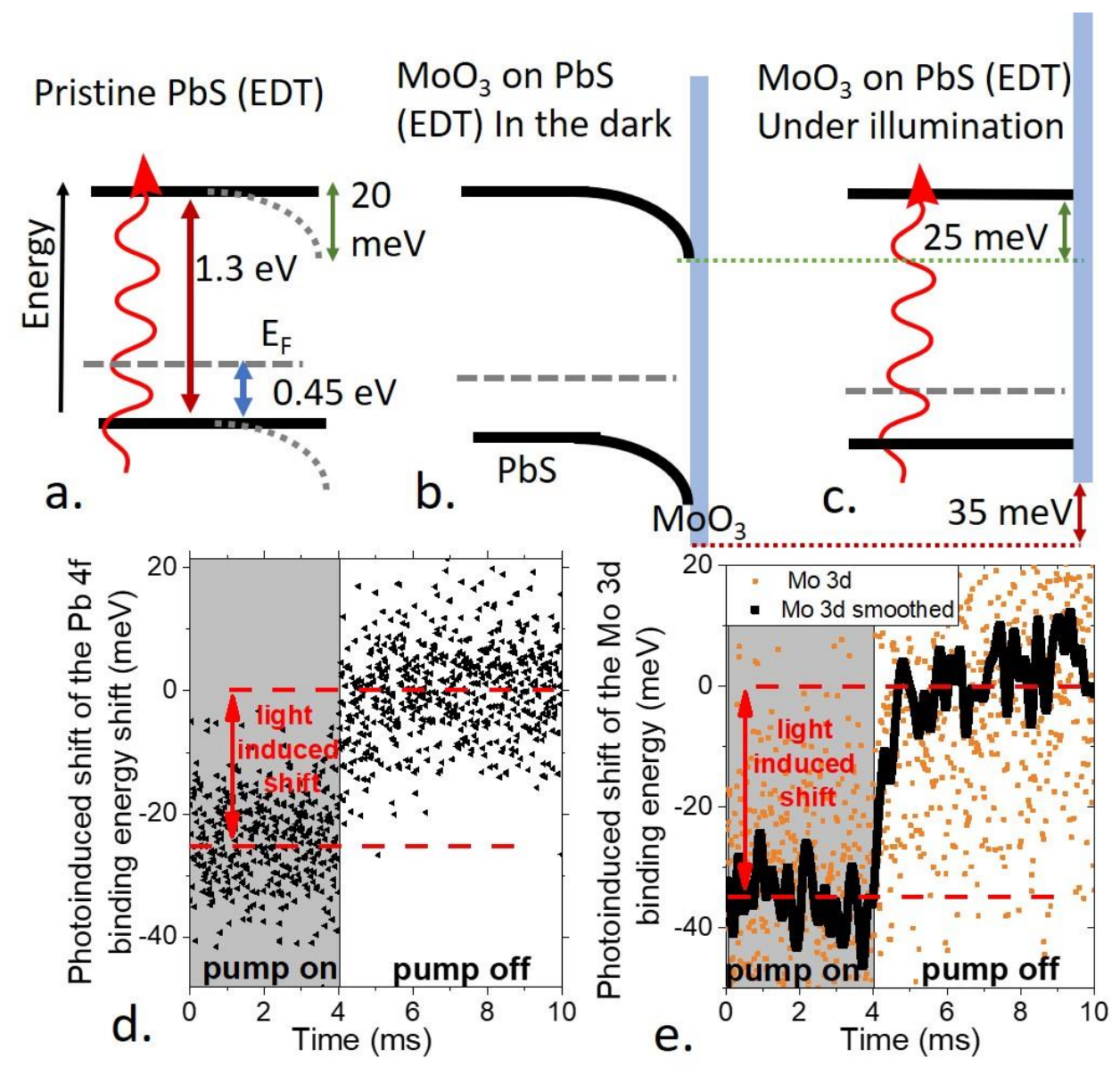

Figure 6: a. Scheme of the band structure of PbS(EDT) CQD layer which band gap can be excited by a photon at $635 \mathrm{~nm}$. The dotted lines highlight the band bending present in the dark. $b$. Scheme 
of the electronic spectrum of a $\mathrm{MoO}_{3}$ layer (blue line on the scheme) coupled with a $\mathrm{PbS}$ (EDT) CQD layer under dark condition. C. Scheme of the electronic spectrum of a $\mathrm{MoO}_{3}$ layer coupled with a $\mathrm{PbS}$ (EDT) CQD layer under excitation by a $635 \mathrm{~nm}$ laser $\mathrm{d}$. Shift of the binding energy of the $\mathrm{Pb} 4 \mathrm{f}$ state from a PbS CQDs (EDT capped) film capped with a $\mathrm{MoO}_{3}$ layer as a function of time, as the laser light at $635 \mathrm{~nm}$ is turned on and off. In part $d$ and e the red lines are used as guide lines for the light induced shift.e. Shift of the binding energy of the $\mathrm{Mo} 3 d$ state from $\mathrm{MoO}_{3}$ layer coupled with a PbS CQD (EDT capped) film as a function of time, as the laser light at $635 \mathrm{~nm}$ is turned on and off. The data smoothing is obtained thanks to a low pass FFT filter.

In the photodiode, the transit time, which is the duration for one carrier to cross the structure, is given by $L^{2} / \mu \mathrm{V}$ with $L$ the device thickness ( $250 \mathrm{~nm}$ for the PbS layer), $\mu$ the carrier mobility and $V$ the applied bias. Assuming a realistic value of $10^{-3} \mathrm{~cm}^{2} \mathrm{~V}^{-1} \mathrm{~s}^{-1}$ for $\mu$ and using $\mathrm{V}=\mathrm{Voc}$ the open circuit voltage of the diode, we can estimate the transit time to be $\approx 1 \mu \mathrm{s}$, indicating that we probe the TRXPS at time scale longer than the characteristic transport time of the structure. What we probe with the TRXPS is the quasi-static relaxation band bending which is the signature of the accumulation of charge at the interface. Here, we observe similar dynamics $(\approx 100 \mu \mathrm{s})$ and shift magnitudes (25 meV for PbS and $35 \mathrm{meV}$ for $\mathrm{MoO}_{3}$ ) for the two layers (see Figure 6d, e and S8), implying that they present a rigid quasi static coupling. This suggests that the depletion width of the nanocrystals at the interface extends into the $\mathrm{MoO}_{3}$ layer and drives its binding energy shift.

\section{CONCLUSION}

To summarize, we have combined static and dynamic photoemissions with transport measurements to provide a coherent description of the electronic structure of a PbS CQD-based solar cell. The $n$ type nature of $\mathrm{I}^{-}$capped PbS CQDs is shown using field-effect transistor measurements and leads to an increase of the $\mathrm{V}_{B}-\mathrm{E}_{F}$ value in static photoemission while we observe a positive shift of the binding energy using TRXPS. TRXPS is then used to selectively probe the dynamics on each side of the $\mathrm{PbS} / \mathrm{MoO}_{3}$ interface. We demonstrate that the $\mathrm{MoO}_{3}$ layer is photosensitized by the presence of the PbS and that the two layers have a similar electrostatic light induced coupling. The hole extraction is identified as a current limitation for the device performance and future design will have to focus on the existing Schottky barrier at this interface. Two directions will be of utmost interest for further understanding of interface coupling and interface dynamics. Firstly, the use of harder X-ray photoemission may inform on energy level alignments at all interfaces of the stack of the solar cell. Secondly, the investigation of the coupling at time scale below the transit time to probe insitu the charge transport at the interface HTL/CQD interface.

\section{ASSOCIATED CONTENT}

The Supporting Information is available free of charge at

Additional information includes additional photoemission core level analysis and TRXPS measurements.

\section{AUTHOR INFORMATION}


The project is supported by ERC starting grant blackQD (grant $n^{\circ} 756225$ ). We acknowledge the use of clean-room facilities from the "Centrale de Proximité Paris-Centre". This work has been supported by the Region Ile-de-France in the framework of DIM Nano-K (grant dopQD). This work was supported by French state funds managed by the ANR within the Investissements d'Avenir programme under reference ANR-11-IDEX-0004-02, and more specifically within the framework of the Cluster of Excellence MATISSE and also by the grant IPER-Nano2 (ANR-18CE30-0023-01), Copin (ANR-19-CE24-0022), Frontal (ANR-19-CE09-0017), Graskop (ANR-19-CE09-0026) and NITQuantum. JQ thanks Chinese Scholar council for PhD grant while AC thanks Agence innovation defense.

\section{REFERENCES}

(1) Konstantatos, G.; Sargent, E. H. Colloidal Quantum Dot Optoelectronics and Photovoltaics; Cambridge University Press, 2013.

(2) Lu, H.; Carroll, G. M.; Neale, N. R.; Beard, M. C. Infrared Quantum Dots: Progress, Challenges, and Opportunities. ACS Nano 2019, 13, 939-953.

(3) McDonald, S. A.; Konstantatos, G.; Zhang, S.; Cyr, P. W.; Klem, E. J. D.; Levina, L.; Sargent, E. H. Solution-Processed PbS Quantum Dot Infrared Photodetectors and Photovoltaics. Nat. Mater. 2005, 4, 138-142.

(4) Lu, K.; Wang, Y.; Liu, Z.; Han, L.; Shi, G.; Fang, H.; Chen, J.; Ye, X.; Chen, S.; Yang, F.; et al. High-Efficiency PbS Quantum-Dot Solar Cells with Greatly Simplified Fabrication Processing via "Solvent-Curing." Adv. Mater. 2018, 30, 1707572.

(5) Semonin, O. E.; Luther, J. M.; Choi, S.; Chen, H.-Y.; Gao, J.; Nozik, A. J.; Beard, M. C. Peak External Photocurrent Quantum Efficiency Exceeding 100\% via MEG in a Quantum Dot Solar Cell. Science 2011, 334, 1530-1533.

(6) Luther, J. M.; Gao, J.; Lloyd, M. T.; Semonin, O. E.; Beard, M. C.; Nozik, A. J. Stability Assessment on a 3\% Bilayer PbS/ZnO Quantum Dot Heterojunction Solar Cell. Adv. Mater. 2010, 22, 3704-3707.

(7) Luther, J. M.; Law, M.; Beard, M. C.; Song, Q.; Reese, M. O.; Ellingson, R. J.; Nozik, A. J. Schottky Solar Cells Based on Colloidal Nanocrystal Films. Nano Lett. 2008, 8, 3488-3492.

(8) Tang, J.; Kemp, K. W.; Hoogland, S.; Jeong, K. S.; Liu, H.; Levina, L.; Furukawa, M.; Wang, X.; Debnath, R.; Cha, D.; et al. Colloidal-Quantum-Dot Photovoltaics Using Atomic-Ligand Passivation. Nat. Mater. 2011, 10, 765-771.

(9) Bederak, D.; Balazs, D. M.; Sukharevska, N. V.; Shulga, A. G.; Abdu-Aguye, M.; Dirin, D. N.; Kovalenko, M. V.; Loi, M. A. Comparing Halide Ligands in PbS Colloidal Quantum Dots for Field-Effect Transistors and Solar Cells. ACS Appl. Nano Mater. 2018, 1, 6882-6889.

(10) Gu, M.; Wang, Y.; Yang, F.; Lu, K.; Xue, Y.; Wu, T.; Fang, H.; Zhou, S.; Zhang, Y.; Ling, X.; et al. Stable PbS Quantum Dot Ink for Efficient Solar Cells by Solution-Phase Ligand Engineering. J. Mater. Chem. A 2019, 7, 15951-15959.

(11) Fischer, A.; Rollny, L.; Pan, J.; Carey, G. H.; Thon, S. M.; Hoogland, S.; Voznyy, O.; Zhitomirsky, D.; Kim, J. Y.; Bakr, O. M.; et al. Directly Deposited Quantum Dot Solids Using a Colloidally Stable Nanoparticle Ink. Adv. Mater. 2013, 25, 5742-5749.

(12) Chuang, C.-H. M.; Brown, P. R.; Bulović, V.; Bawendi, M. G. Improved Performance and Stability in Quantum Dot Solar Cells through Band Alignment Engineering. Nat. Mater. 2014, 13, 796-801.

(13) Brown, P. R.; Kim, D.; Lunt, R. R.; Zhao, N.; Bawendi, M. G.; Grossman, J. C.; Bulović, V. Energy Level Modification in Lead Sulfide Quantum Dot Thin Films through Ligand Exchange. ACS Nano 2014, 8, 5863-5872.

(14) Ganesan, A. A.; Houtepen, A. J.; Crisp, R. W. Quantum Dot Solar Cells: Small Beginnings Have Large Impacts. Appl. Sci. 2018, 8, 1867. 
(15) Miller, E. M.; Kroupa, D. M.; Zhang, J.; Schulz, P.; Marshall, A. R.; Kahn, A.; Lany, S.; Luther, J. M.; Beard, M. C.; Perkins, C. L.; et al. Revisiting the Valence and Conduction Band Size Dependence of PbS Quantum Dot Thin Films. ACS Nano 2016, 10, 3302-3311.

(16) Kroupa, D. M.; Vörös, M.; Brawand, N. P.; McNichols, B. W.; Miller, E. M.; Gu, J.; Nozik, A. J.; Sellinger, A.; Galli, G.; Beard, M. C. Tuning Colloidal Quantum Dot Band Edge Positions through Solution-Phase Surface Chemistry Modification. Nat. Commun. 2017, 8, 15257.

(17) Martinez, B.; Livache, C.; Notemgnou Mouafo, L. D.; Goubet, N.; Keuleyan, S.; Cruguel, H.; Ithurria, S.; Aubin, H.; Ouerghi, A.; Doudin, B.; et al. HgSe Self-Doped Nanocrystals as a Platform to Investigate the Effects of Vanishing Confinement. ACS Appl. Mater. Interfaces 2017, 9, 36173-36180.

(18) Martinez, B.; Plamont, R.; Gréboval, C.; Rastogi, P.; Prado, Y.; Qu, J.; Chu, A.; Livache, C.; Xu, X. Z.; Cruguel, H.; et al. Azobenzenes as Light-Activable Carrier Density Switches in Nanocrystals. J. Phys. Chem. C 2019, 123, 27257-27263.

(19) Nugraha, M. I.; Matsui, H.; Bisri, S. Z.; Sytnyk, M.; Heiss, W.; Loi, M. A.; Takeya, J. Tunable Doping in PbS Nanocrystal Field-Effect Transistors Using Surface Molecular Dipoles. APL Mater. 2016, 4, 116105.

(20) Ramade, J.; Qu, J.; Chu, A.; Gréboval, C.; Livache, C.; Goubet, N.; Martinez, B.; Vincent, G.; Lhuillier, E. Potential of Colloidal Quantum Dot Based Solar Cells for Near-Infrared Active Detection. ACS Photonics 2020, 7, 272-278.

(21) Konstantatos, G.; Howard, I.; Fischer, A.; Hoogland, S.; Clifford, J.; Klem, E.; Levina, L.; Sargent, E. H. Ultrasensitive Solution-Cast Quantum Dot Photodetectors. Nature 2006, 442, 180-183.

(22) Konstantatos, G.; Sargent, E. H. PbS Colloidal Quantum Dot Photoconductive Photodetectors: Transport, Traps, and Gain. Appl. Phys. Lett. 2007, 91, 173505.

(23) Rauch, T.; Böberl, M.; Tedde, S. F.; Fürst, J.; Kovalenko, M. V.; Hesser, G.; Lemmer, U.; Heiss, W.; Hayden, O. Near-Infrared Imaging with Quantum-Dot-Sensitized Organic Photodiodes. Nat. Photonics 2009, 3, 332-336.

(24) Georgitzikis, E.; Malinowski, P. E.; Li, Y.; Maes, J.; Hagelsieb, L. M.; Guerrieri, S.; Hens, Z.; Heremans, P.; Cheyns, D. Integration of PbS Quantum Dot Photodiodes on Silicon for NIR Imaging. IEEE Sens. J. 2019, 6841-6848.

(25) Chernomordik, B. D.; Marshall, A. R.; Pach, G. F.; Luther, J. M.; Beard, M. C. Quantum Dot Solar Cell Fabrication Protocols. Chem. Mater. 2017, 29, 189-198.

(26) Neppl, S.; Gessner, O. Time-Resolved X-Ray Photoelectron Spectroscopy Techniques for the Study of Interfacial Charge Dynamics. J. Electron Spectrosc. Relat. Phenom. 2015, 200, 6477.

(27) Bergeard, N.; Silly, M. G.; Krizmancic, D.; Chauvet, C.; Guzzo, M.; Ricaud, J. P.; Izquierdo, M.; Stebel, L.; Pittana, P.; Sergo, R.; et al. Time-Resolved Photoelectron Spectroscopy Using Synchrotron Radiation Time Structure. J. Synchrotron Radiat. 2011, 18, 245-250.

(28) Spencer, B. F.; Cliffe, M. J.; Graham, D. M.; Hardman, S. J. O.; Seddon, E. A.; Syres, K. L.; Thomas, A. G.; Sirotti, F.; Silly, M. G.; Akhtar, J.; et al. Chemically-Specific Time-Resolved Surface Photovoltage Spectroscopy: Carrier Dynamics at the Interface of Quantum Dots Attached to a Metal Oxide. Surf. Sci. 2015, 641, 320-325.

(29) Livache, C.; Izquierdo, E.; Martinez, B.; Dufour, M.; Pierucci, D.; Keuleyan, S.; Cruguel, H.; Becerra, L.; Fave, J. L.; Aubin, H.; et al. Charge Dynamics and Optolectronic Properties in HgTe Colloidal Quantum Wells. Nano Lett. 2017, 17, 4067-4074.

(30) Spencer, B. F.; Leontiadou, M. A.; Clark, P. C. J.; Williamson, A. I.; Silly, M. G.; Sirotti, F.; Fairclough, S. M.; Tsang, S. C. E.; Neo, D. C. J.; Assender, H. E.; et al. Charge Dynamics at Heterojunctions for PbS/ZnO Colloidal Quantum Dot Solar Cells Probed with Time-Resolved Surface Photovoltage Spectroscopy. Appl. Phys. Lett. 2016, 108, 091603.

(31) Gréboval, C.; Izquierdo, E.; Livache, C.; Martinez, B.; Dufour, M.; Goubet, N.; Moghaddam, N.; Qu, J.; Chu, A.; Ramade, J.; et al. Impact of Dimensionality and Confinement on the Electronic Properties of Mercury Chalcogenide Nanocrystals. Nanoscale 2019, 11, 39053915.

(32) Amelot, D.; Rastogi, P.; Martinez, B.; Gréboval, C.; Livache, C.; Bresciani, F. A.; Qu, J.; Chu, A.; Goyal, M.; Chee, S.-S.; et al. Revealing the Band Structure of FAPI Quantum Dot Film and 
Its Interfaces with Electron and Hole Transport Layer Using Time Resolved Photoemission. J. Phys. Chem. C 2020, 124, 3873-3880.

(33) Hazama, Y.; Ishida, Y.; Zhu, L.; Kim, C.; Shin, S.; Akiyama, H. Revealing Solar-Cell Photovoltage Dynamics at the Picosecond Time Scale with Time-Resolved Photoemission Spectroscopy. Phys. Rev. Appl. 2018, 10, 034056.

(34) Ogawa, M.; Yamamoto, S.; Fujikawa, K.; Hobara, R.; Yukawa, R.; Yamamoto, Sh.; Kitagawa, S.; Pierucci, D.; Silly, M. G.; Lin, C.-H.; et al. Relaxations of the Surface Photovoltage Effect on the Atomically Controlled Semiconductor Surfaces Studied by Time-Resolved Photoemission Spectroscopy. Phys. Rev. B 2013, 88, 165313.

(35) Ishida, Y.; Togashi, T.; Yamamoto, K.; Tanaka, M.; Kiss, T.; Otsu, T.; Kobayashi, Y.; Shin, S. Time-Resolved Photoemission Apparatus Achieving Sub-20-MeV Energy Resolution and High Stability. Rev. Sci. Instrum. 2014, 85, 123904.

(36) Hines, M. A.; Scholes, G. D. Colloidal PbS Nanocrystals with Size-Tunable Near-Infrared Emission: Observation of Post-Synthesis Self-Narrowing of the Particle Size Distribution. Adv. Mater. 2003, 15, 1844-1849.

(37) Moreels, I.; Lambert, K.; Smeets, D.; De Muynck, D.; Nollet, T.; Martins, J. C.; Vanhaecke, F.; Vantomme, A.; Delerue, C.; Allan, G.; et al. Size-Dependent Optical Properties of Colloidal PbS Quantum Dots. ACS Nano 2009, 3, 3023-3030.

(38) Nag, A.; Kovalenko, M. V.; Lee, J.-S.; Liu, W.; Spokoyny, B.; Talapin, D. V. Metal-Free Inorganic Ligands for Colloidal Nanocrystals: $\mathrm{S}^{2-}, \mathrm{HS}^{-}, \mathrm{Se}^{2-}, \mathrm{HSe}^{-}, \mathrm{Te}^{2-}, \mathrm{HTe}^{-}, \mathrm{TeS}_{3}{ }^{2-}, \mathrm{OH}^{-}$, and $\mathrm{NH}_{2}{ }^{-}$as Surface Ligands. J. Am. Chem. Soc. 2011, 133, 10612-10620.

(39) Kim, T. G.; Choi, H.; Jeong, S.; Kim, J. W. Electronic Structure of PbS Colloidal Quantum Dots on Indium Tin Oxide and Titanium Oxide. J. Phys. Chem. C 2014, 118, 27884-27889.

(40) Garcia-Gutierrez, D. F.; Hernandez-Casillas, L. P.; Cappellari, M. V.; Fungo, F.; MartínezGuerra, E.; García-Gutiérrez, D. I. Influence of the Capping Ligand on the Band Gap and Electronic Levels of PbS Nanoparticles through Surface Atomistic Arrangement Determination. ACS Omega 2018, 3, 393-405.

(41) Gleason-Rohrer, D. C.; Brunschwig, B. S.; Lewis, N. S. Measurement of the Band Bending and Surface Dipole at Chemically Functionalized Si(111)/Vacuum Interfaces. J. Phys. Chem. C 2013, 117, 18031-18042.

(42) Cademartiri, L.; Montanari, E.; Calestani, G.; Migliori, A.; Guagliardi, A.; Ozin, G. A. SizeDependent Extinction Coefficients of PbS Quantum Dots. J. Am. Chem. Soc. 2006, 128, 10337-10346.

(43) Tahara, H.; Sakamoto, M.; Teranishi, T.; Kanemitsu, Y. Quantum Coherence of Multiple Excitons Governs Absorption Cross-Sections of PbS/CdS Core/Shell Nanocrystals. Nat. Commun. 2018, 9, 3179.

(44) Lhuillier, E.; Ithurria, S.; Descamps-Mandine, A.; Douillard, T.; Castaing, R.; Xu, X. Z.; Taberna, P.-L.; Simon, P.; Aubin, H.; Dubertret, B. Investigating the N- and p-Type Electrolytic Charging of Colloidal Nanoplatelets. J. Phys. Chem. C 2015, 119, 21795-21799.

(45) King, L. A.; Parkinson, B. A. Photosensitization of ZnO Crystals with lodide-Capped PbSe Quantum Dots. J. Phys. Chem. Lett. 2016, 7, 2844-2848.

(46) Mali, S. S.; Desai, S. K.; Kalagi, S. S.; Betty, C. A.; Bhosale, P. N.; Devan, R. S.; Ma, Y.-R. R.; Patil, P. S. PbS Quantum Dot Sensitized Anatase $\mathrm{TiO}_{2}$ Nanocorals for Quantum DotSensitized Solar Cell Applications. Dalton Trans. 2012, 41, 6130-6136.

(47) Arion, T.; Neppl, S.; Roth, F.; Shavorskiy, A.; Bluhm, H.; Hussain, Z.; Gessner, O.; Eberhardt, W. Site-Specific Probing of Charge Transfer Dynamics in Organic Photovoltaics. Appl. Phys. Lett. 2015, 106, 121602.

(48) Hu, L.; Mandelis, A.; Lan, X.; Melnikov, A.; Hoogland, S.; Sargent, E. H. Imbalanced Charge Carrier Mobility and Schottky Junction Induced Anomalous Current-Voltage Characteristics of Excitonic PbS Colloidal Quantum Dot Solar Cells. Sol. Energy Mater. Sol. Cells 2016, 155, 155-165.

(49) Chuang, C.-H. M.; Maurano, A.; Brandt, R. E.; Hwang, G. W.; Jean, J.; Buonassisi, T.; Bulović, V.; Bawendi, M. G. Open-Circuit Voltage Deficit, Radiative Sub-Bandgap States, and Prospects in Quantum Dot Solar Cells. Nano Lett. 2015, 15, 3286-3294. 
(50) Pradhan, S.; Stavrinadis, A.; Gupta, S.; Christodoulou, S.; Konstantatos, G. Breaking the Open-Circuit Voltage Deficit Floor in PbS Quantum Dot Solar Cells through Synergistic Ligand and Architecture Engineering. ACS Energy Lett. 2017, 2, 1444-1449.

(51) Kim, G.-H.; García de Arquer, F. P.; Yoon, Y. J.; Lan, X.; Liu, M.; Voznyy, O.; Krishnan Jagadamma, L.; Saud Abbas, A.; Yang, Z.; Fan, F.; et al. Correction to High-Efficiency Colloidal Quantum Dot Photovoltaics via Robust Self-Assembled Monolayers. Nano Lett. 2016, 16, 822-822.

(52) Kramer, I. J.; Levina, L.; Debnath, R.; Zhitomirsky, D.; Sargent, E. H. Solar Cells Using Quantum Funnels. Nano Lett. 2011, 11, 3701-3706. 


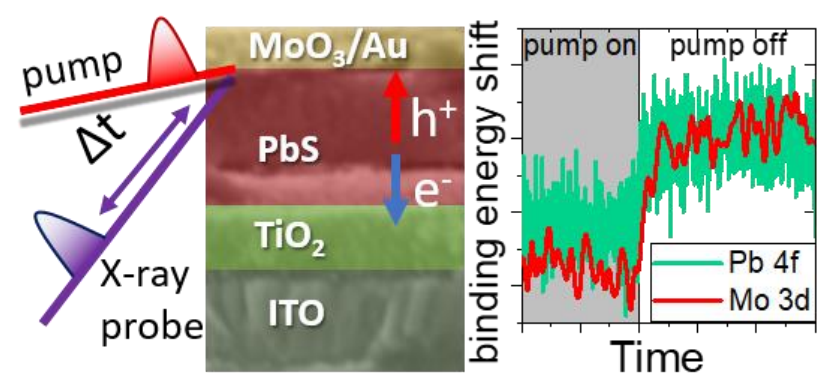

